\title{
Heinz-type mean curvature estimates in Lorentz-Minkowski space
}

\author{
Atsufumi Honda $^{1} \cdot$ Yu Kawakami ${ }^{2}$ (D) Miyuki Koiso ${ }^{3} \cdot$ Syunsuke Tori $^{4}$
}

Received: 15 April 2020 / Accepted: 23 September 2020 / Published online: 8 October 2020

(C) The Author(s) 2020

\begin{abstract}
We provide a unified description of Heinz-type mean curvature estimates under an assumption on the gradient bound for space-like graphs and time-like graphs in the Lorentz-Minkowski space. As a corollary, we give a unified vanishing theorem of mean curvature for these entire graphs of constant mean curvature.
\end{abstract}

Keywords Heinz-type mean curvature estimate - Bernstein-type theorem · Space-like graph · Time-like graph · Constant mean curvature

Mathematics Subject Classification Primary 53A10; Secondary 53B30 - 53C24 • $53 \mathrm{C} 42$

Dedicated to Professor Atsushi Kasue on the occasion of his 65th birthday

The authors were partially supported by JSPS KAKENHI Grant Number JP18H04487, JP19K03463, JP19K14526, JP20H04642 and JP20H01801.

$\bowtie \quad$ Yu Kawakami

y-kwkami@se.kanazawa-u.ac.jp

Atsufumi Honda

honda-atsufumi-kp@ynu.ac.jp

Miyuki Koiso

koiso@math.kyushu-u.ac.jp

Syunsuke Tori

shunsuke-1350@stu.kanazawa-u.ac.jp

1 Department of Applied Mathematics, Faculty of Engineering, Yokohama National University, 79-5, Tokiwadai, Hodogaya-ku, Yokohama 240-8501, Japan

2 Institute of Science and Engineering, Faculty of Mathematics and Physics, Kanazawa University, Kanazawa 920-1192, Japan

3 Institute of Mathematics for Industry, Kyushu University, 744, Motooka Nishi-ku, Fukuoka 819-0395, Japan

4 Graduate School of Natural Science and Technology, Kanazawa University, Kanazawa 920-1192, Japan 


\section{Introduction}

The celebrated theorem of Bernstein [1] (as for a simple proof, see [2]), stating that the only entire minimal graph in the Euclidean 3 -space $\mathbf{R}^{3}$ is a plane, has played a seminal role in the evolution of surface theory. For instance, by using this theorem, we can show the uniqueness theorem for an entire graph of constant mean curvature in $\mathbf{R}^{3}$. Indeed Heinz [3] proved that if $\varphi\left(u^{1}, u^{2}\right)$ is a $C^{2}$-differentiable function defined on the open disk $B^{2}(R)$ with center at the origin and radius $R(>0)$ in the $\left(u^{1}, u^{2}\right)$-plane, and the mean curvature $H$ of the graph $\Gamma_{\varphi}:=\left\{\left(u^{1}, u^{2}, \varphi\left(u^{1}, u^{2}\right)\right) \in \mathbf{R}^{3} ;\left(u^{1}, u^{2}\right) \in B^{2}(R)\right\}$ of $\varphi$ satisfies $|H| \geq \alpha>0$, where $\alpha$ is constant, then $R \leq 1 / \alpha$. As a corollary, we have a vanishing theorem of mean curvature for entire graphs of constant mean curvature, that is, if $\Gamma_{\varphi}$ is an entire graph of constant mean curvature, then $H \equiv 0$. Combining these results, we obtain that the only entire graph of constant mean curvature in $\mathbf{R}^{3}$ is a plane (see [4, Section 2.1]). The Heinz result was extended to the case of graphic hypersurfaces in the Euclidean $(n+1)$-space $\mathbf{R}^{n+1}$ by Chern [5] and Flanders [6].

We will study this subject for graphic hypersurfaces in the Lorentz-Minkowski space. Here we recall some basic definitions and fundamental facts. We denote by $\mathbf{R}_{1}^{n+1}=\left(\mathbf{R}^{n+1},\langle,\rangle_{L}\right)$ the Lorentz-Minkowski $(n+1)$-space with the Lorentzian metric

$$
\left\langle\left(x^{1}, \ldots, x^{n}, x^{n+1}\right),\left(y^{1}, \ldots, y^{n}, y^{n+1}\right)\right\rangle_{L}:=x^{1} y^{1}+\cdots+x^{n} y^{n}-x^{n+1} y^{n+1},
$$

where $\left(x^{1}, \ldots, x^{n}, x^{n+1}\right),\left(y^{1}, \ldots, y^{n}, y^{n+1}\right) \in \mathbf{R}^{n+1}$. Let $M^{n}$ be a smooth orientable $n$-manifold. An immersion $f: M^{n} \rightarrow \mathbf{R}_{1}^{n+1}$ is called space-like if the induced metric $g:=f^{*}\langle,\rangle_{L}$ on $M^{n}$ is Riemannian, and is called time-like if $g$ on $M^{n}$ is Lorentzian. A space-like (resp. time-like) immersion of constant mean curvature is called a spacelike (resp. time-like) constant mean curvature immersion. In particular, a space-like immersion with vanishing mean curvature is called a space-like maximal immersion and a time-like immersion with vanishing mean curvature is called a time-like minimal immersion.

Let $\psi: \Omega \rightarrow \mathbf{R}$ be a $C^{2}$-differentiable function defined on a domain $\Omega$ in $\mathbf{R}^{n}$. We consider the graph $\Gamma_{\psi}:=\left\{\left(u^{1}, \ldots, u^{n}, \psi\left(u^{1}, \ldots, u^{n}\right)\right) \in \mathbf{R}_{1}^{n+1} ;\left(u^{1}, \ldots, u^{n}\right) \in \Omega\right\}$ of $\psi$. If $\Omega=\mathbf{R}^{n}$, then $\Gamma_{\psi}$ is called an entire graph of $\psi$ in $\mathbf{R}_{1}^{n+1}$. If the graph $\Gamma_{\psi}$ of $\psi$ is space-like, the gradient $\nabla \psi:=\left(\psi_{u^{1}}, \ldots, \psi_{u^{n}}\right)$ of $\psi$ satisfies $|\nabla \psi|:=$ $\sqrt{\left(\psi_{u^{1}}\right)^{2}+\cdots+\left(\psi_{u^{n}}\right)^{2}}<1$ on $\Omega$, and

$$
n H=\sum_{i=1}^{n} \frac{\partial}{\partial u^{i}}\left(\frac{\psi_{u^{i}}}{\sqrt{1-|\nabla \psi|^{2}}}\right)=\operatorname{div}\left(\frac{\nabla \psi}{\sqrt{1-|\nabla \psi|^{2}}}\right),
$$

that is,

$$
\left(1-|\nabla \psi|^{2}\right) \sum_{i=1}^{n} \frac{\partial^{2} \psi}{\left(\partial u^{i}\right)^{2}}+\sum_{i, j=1}^{n} \frac{\partial \psi}{\partial u^{i}} \frac{\partial \psi}{\partial u^{j}} \frac{\partial^{2} \psi}{\partial u^{i} \partial u^{j}}=n H\left(1-|\nabla \psi|^{2}\right)^{3 / 2}
$$


holds. Here $H$ is the mean curvature of $\Gamma_{\psi}$ and $\psi_{u^{i}}:=\partial \psi / \partial u^{i}$. If the graph $\Gamma_{\psi}$ of $\psi$ is time-like, its gradient $\nabla \psi$ satisfies $|\nabla \psi|>1$ on $\Omega$, and

$$
n H=\sum_{i=1}^{n} \frac{\partial}{\partial u^{i}}\left(\frac{\psi_{u^{i}}}{\sqrt{|\nabla \psi|^{2}-1}}\right)=\operatorname{div}\left(\frac{\nabla \psi}{\sqrt{|\nabla \psi|^{2}-1}}\right)
$$

holds.

For entire space-like maximal graphs in $\mathbf{R}_{1}^{n+1}$, the following uniqueness theorem, called the Calabi-Bernstein theorem, is well-known. This result was proved by Calabi [7] for $n \leq 4$ and Cheng-Yau [8] for all $n$.

Fact 1.1 (The Calabi-Bernstein theorem) Any entire space-like maximal graph is a space-like hyperplane in $\mathbf{R}_{1}^{n+1}$.

We remark that an improvement of this theorem and a fluid mechanical interpretation of the duality between minimal graphs in $\mathbf{R}^{3}$ and space-like maximal graphs in $\mathbf{R}_{1}^{3}$ are discussed in [9] (cf. [10]).

On the other hand, for entire space-like constant mean curvature graphs, the uniqueness theorem does not hold in general. In fact, the graph of the function

$$
\psi\left(u^{1}, \ldots, u^{n}\right)=\left(\left(u^{1}\right)^{2}+\cdots+\left(u^{n}\right)^{2}+\frac{1}{H^{2}}\right)^{1 / 2} \quad(H>0)
$$

is an entire space-like constant mean curvature $H$ graph which is not a hyperplane. This graph is a hyperboloid. Many other entire space-like constant mean curvature graphs are constructed by Treibergs [11]. Hence the following questions naturally arise. What are the situations where Heinz-type mean curvature estimate and Bernstein-type uniqueness theorem hold for space-like graphs in $\mathbf{R}_{1}^{n+1}$ ? What are these situations for time-like graphs in $\mathbf{R}_{1}^{n+1}$ ?

In this paper, we perform a systematic study for these questions. In particular, we give a unified description of Heinz-type mean curvature estimates under an assumption on the gradient bound for space-like graphs and time-like graphs in $\mathbf{R}_{1}^{n+1}$ (Theorem 2.1). As a corollary, we obtain a unified vanishing theorem of mean curvature for entire graphs of constant mean curvature with a gradient bound (Corollary 2.4). In Sect. 3, by applying these results, we answer the questions mentioned above. Moreover we give a short commentary about the recent study by the authors for entire constant mean curvature graphs in $\mathbf{R}_{1}^{n+1}$.

Finally, the authors gratefully acknowledge the useful comments from Shintaro Akamine and Atsushi Kasue during the preparation of this paper.

\section{Main results}

The main theorem is stated as follows: 
Theorem 2.1 Let $B^{n}(R)$ be the open $n$-ball with center at the origin and radius $R(>0)$ in $\mathbf{R}^{n}$, and $\psi\left(u^{1}, \ldots, u^{n}\right)$ a $C^{2}$-differentiable function on $B^{n}(R)$. Suppose that there exist constants $M>0$ and $k \in \mathbf{R}$ such that

$$
\frac{|\nabla \psi|}{\sqrt{\left.|1-| \nabla \psi\right|^{2} \mid}} \leq M\left(\left(u^{1}\right)^{2}+\cdots+\left(u^{n}\right)^{2}\right)^{k}
$$

on $B^{n}(R)$. Assume that $\Gamma_{\psi}$ is a space-like or time-like graph of $\psi$ in $\mathbf{R}_{1}^{n+1}$. If the mean curvature $H$ of $\Gamma_{\psi}$ satisfies the inequality

$$
|H| \geq \alpha>0
$$

where $\alpha$ is constant, then the following inequality holds:

$$
\alpha \leq M R^{2 k-1}
$$

Proof Let $\omega$ be the $(n-1)$-form given by

$$
\omega=\sum_{i=1}^{n}(-1)^{i-1}\left(\frac{\psi_{u^{i}}}{\sqrt{\left.|1-| \nabla \psi\right|^{2} \mid}}\right) d u^{1} \wedge \cdots \wedge \widehat{d u^{i}} \wedge \cdots \wedge d u^{n}
$$

where $\widehat{d u^{i}}$ means that $d u^{i}$ is omitted from the wedge product. Then we can obtain $d \omega=n H d u^{1} \wedge \cdots \wedge d u^{n}$ by (1) and (2). Take any positive number $R^{\prime}$ satisfying $0<R^{\prime}<R$. The Stokes theorem yields the following equality:

$$
\int_{\bar{B}^{n}\left(R^{\prime}\right)} d \omega=\int_{\partial B^{n}\left(R^{\prime}\right)} \omega
$$

where $\bar{B}^{n}\left(R^{\prime}\right)$ is the closure and $\partial B^{n}\left(R^{\prime}\right)$ is the boundary of $B^{n}\left(R^{\prime}\right)$.

We may assume $H \geq \alpha>0$ by changing the direction of the normal vector if necessary. Let $V_{n}$ be the volume of the unit closed $n$-ball $\bar{B}^{n}(1)$ and $A_{n-1}$ the volume of the unit $(n-1)$-sphere $\partial B^{n}(1)$. Then, by radial integration, $n V_{n}=A_{n-1}$ holds. The absolute value of the left-hand side of (7) becomes

$$
\begin{aligned}
\left|\int_{\bar{B}^{n}\left(R^{\prime}\right)} d \omega\right| & =n \int_{\bar{B}^{n}\left(R^{\prime}\right)} H d u^{1} \cdots d u^{n} \\
& \geq n \alpha \int_{\bar{B}^{n}\left(R^{\prime}\right)} d u^{1} \cdots d u^{n}=n \alpha\left(R^{\prime}\right)^{n} V_{n}
\end{aligned}
$$

On the other hand, by using the Cauchy-Schwarz inequality, the absolute value of the right-hand side of (7) becomes

$$
\left|\int_{\partial B^{n}\left(R^{\prime}\right)} \omega\right|=\left|\int_{\partial B^{n}\left(R^{\prime}\right)} \sum_{i=1}^{n}(-1)^{i-1}\left(\frac{\psi_{u^{i}}}{\sqrt{\left.|1-| \nabla \psi\right|^{2} \mid}}\right) d u^{1} \cdots \widehat{d u^{i}} \cdots d u^{n}\right|
$$




$$
\leq \int_{\partial B^{n}\left(R^{\prime}\right)} \frac{|\nabla \psi|}{\sqrt{\left.|1-| \nabla \psi\right|^{2} \mid}}\left(\sum_{i=1}^{n}\left(d u^{1} \cdots \widehat{d u^{i}} \cdots d u^{n}\right)^{2}\right)^{1 / 2}
$$

By (4), we have

$$
\begin{aligned}
\left|\int_{\partial B^{n}\left(R^{\prime}\right)} \omega\right| & \leq M\left(R^{\prime}\right)^{2 k} \int_{\partial B^{n}\left(R^{\prime}\right)}\left(\sum_{i=1}^{n}\left(d u^{1} \cdots \widehat{d u^{i}} \cdots d u^{n}\right)^{2}\right)^{1 / 2} \\
& =M\left(R^{\prime}\right)^{n+2 k-1} A_{n-1} .
\end{aligned}
$$

We thus obtain $n \alpha\left(R^{\prime}\right)^{n} V_{n} \leq M\left(R^{\prime}\right)^{n+2 k-1} A_{n-1}$, that is, $\alpha \leq M\left(R^{\prime}\right)^{2 k-1}$. The proof is completed by letting $R^{\prime} \rightarrow R$.

From the above argument, we give the following generalization of the result which was obtained by Salavessa [12, Theorem 1.5] for space-like graphs in $\mathbf{R}_{1}^{n+1}$.

Proposition 2.2 Let $D$ be a relatively compact domain (i.e. its closure $\bar{D}$ is compact) of $\mathbf{R}^{n}$ with smooth boundary $\partial D$. Assume that $\Gamma_{\psi} \subset \mathbf{R}_{1}^{n+1}$ is a space-like or time-like graph of a $C^{2}$-differentiable function $\psi$. Set $m_{D}:=\max _{D}|\nabla \psi|$ if $\Gamma_{\psi}$ is a space-like graph in $\mathbf{R}_{1}^{n+1}$ and $m_{D}:=\min _{\bar{D}}|\nabla \psi|$ if $\Gamma_{\psi}$ is a time-like graph in $\mathbf{R}_{1}^{n+1}$. Then, for the mean curvature $H$ of $\Gamma_{\psi}$, we have

$$
\min _{\bar{D}}|H| \leq \frac{1}{n} \frac{m_{D}}{\sqrt{\left|1-\left(m_{D}\right)^{2}\right|}} \frac{A(\partial D)}{V(\bar{D})} .
$$

Here $V(\bar{D})($ resp. $A(\partial D))$ is the volume of $\bar{D}$ (resp. $\partial D)$.

Remark 2.3 To be precise, in [12, Theorem 1.5] Salavessa obtained this inequality for space-like graphs of $M \times N$, where $M$ is a Riemannian $n$-manifold and $N$ is an oriented Lorentzian 1-manifold.

Indeed, from the Stokes theorem for the $(n-1)$-form $\omega$ given by $(6)$, we obtain

$$
\int_{\bar{D}} d \omega=\int_{\partial D} \omega
$$

Then the absolute value of the left-hand side of (9) becomes

$$
\left|\int_{\bar{D}} d \omega\right| \geq n \min _{\bar{D}}|H| V(\bar{D}) .
$$

Since the function $t / \sqrt{1-t^{2}}$ is monotone increasing on $0<t<1$ and $t / \sqrt{t^{2}-1}$ is monotone decreasing on $t>1$, we have

$$
\left|\int_{\partial D} \omega\right| \leq \frac{m_{D}}{\sqrt{\left|1-\left(m_{D}\right)^{2}\right|}} A(\partial D) .
$$

We have thus proved the inequality (8). 
As a corollary of Theorem 2.1, we give the following unified vanishing theorem of mean curvature for entire space-like graphs and time-like graphs of constant mean curvature in $\mathbf{R}_{1}^{n+1}$.

Corollary 2.4 Assume that $\Gamma_{\psi}$ is an entire space-like or time-like graph of a $C^{2}$ differentiable function $\psi\left(u^{1}, \ldots, u^{n}\right)$ in $\mathbf{R}_{1}^{n+1}$. If the entire graph $\Gamma_{\psi}$ has constant mean curvature and there exist constants $M>0$ and $\varepsilon>0$ such that

$$
\frac{|\nabla \psi|}{\sqrt{\left.|1-| \nabla \psi\right|^{2} \mid}} \leq M\left(\left(u^{1}\right)^{2}+\cdots+\left(u^{n}\right)^{2}\right)^{(1 / 2)-\varepsilon}
$$

on $\mathbf{R}^{n}$, then its mean curvature must vanish everywhere.

Proof By Theorem 2.1, the mean curvature $H$ of $\Gamma_{\psi}$ satisfies

$$
|H| \leq \frac{M}{R^{2 \varepsilon}}
$$

on $B^{n}(R)$. We obtain $H \equiv 0$ by letting $R \rightarrow+\infty$.

\section{Applications}

\subsection{Space-like case}

Then $|\nabla \psi|<1$ holds. We give a geometric interpretation for $|\nabla \psi| / \sqrt{1-|\nabla \psi|^{2}}$. When the graph $\Gamma_{\psi}$ is space-like,

$$
v\left(=v\left(u^{1}, \cdots, u^{n}\right)\right)=\frac{1}{\sqrt{1-|\nabla \psi|^{2}}}\left(\psi_{u^{1}}, \cdots, \psi_{u^{n}}, 1\right)
$$

is the time-like unit normal vector field $\nu$ of $\Gamma_{\psi}$. Since $e_{n+1}=(0, \cdots, 0,1) \in \mathbf{R}_{1}^{n+1}$ is also time-like, there exists a unique real-valued function $\theta\left(=\theta\left(u^{1}, \cdots, u^{n}\right)\right) \geq 0$ such that $\left\langle v, e_{n+1}\right\rangle_{L}=-\cosh \theta$. This function $\theta$ is called the hyperbolic angle between $v$ and $e_{n+1}$ (see [13]). By simple calculation, we have

$$
\sinh \theta=\frac{|\nabla \psi|}{\sqrt{1-|\nabla \psi|^{2}}}
$$

We remark that López [14] studies the function $|\nabla \psi| / \sqrt{1-|\nabla \psi|^{2}}$ from the viewpoint of the Dirichlet problem.

From Theorem 2.1, we obtain the following Heinz-type mean curvature estimate. 
Corollary 3.1 Let $\Gamma_{\psi}$ be a space-like graph of a $C^{2}$-differentiable function $\psi\left(u^{1}, \ldots, u^{n}\right)$ on $B^{n}(R)$ in $\mathbf{R}_{1}^{n+1}$ and $\theta$ the hyperbolic angle between $v$ and $e_{n+1}$. Suppose that there exist $M>0$ and $k \in \mathbf{R}$ such that

$$
\sinh \theta \leq M\left(\left(u^{1}\right)^{2}+\cdots+\left(u^{n}\right)^{2}\right)^{k}
$$

on $B^{n}(R)$. If the mean curvature $H$ of $\Gamma_{\psi}$ satisfies the inequality $|H| \geq \alpha>0$, where $\alpha$ is constant, then $\alpha \leq M R^{2 k-1}$ holds.

By virtue of Fact 1.1 and Corollary 2.4, we obtain the following Bernstein-type theorem for entire space-like constant mean curvature graphs in $\mathbf{R}_{1}^{n+1}$.

Corollary 3.2 If an entire space-like graph $\Gamma_{\psi}$ of a $C^{2}$-differentiable function $\psi\left(u^{1}, \ldots, u^{n}\right)$ in $\mathbf{R}_{1}^{n+1}$ has constant mean curvature and there exist constants $M>0$ and $\varepsilon>0$ such that

$$
\sinh \theta \leq M\left(\left(u^{1}\right)^{2}+\cdots+\left(u^{n}\right)^{2}\right)^{(1 / 2)-\varepsilon}
$$

on $\mathbf{R}^{n}$, then it must be a space-like hyperplane. Here $\theta$ is the hyperbolic angle between $v$ and $e_{n+1}$.

Corollary 3.2 is optimal because there exists an example which is not congruent to a space-like hyperplane and satisfies (14) for $\varepsilon=0$. In fact, the function $\psi$ given by (3) is not linear and its graph has constant mean curvature. Moreover it satisfies

$$
\sinh \theta=H \sqrt{\left(u^{1}\right)^{2}+\cdots+\left(u^{n}\right)^{2}},
$$

which is the equality in (14) for $\varepsilon=0$.

From the case where $\varepsilon=1 / 2$ in Corollary 3.2, we obtain the following Liouvilletype result.

Corollary 3.3 Let $\Gamma_{\psi}$ be an entire space-like constant mean curvature graph of a $C^{2}$ differentiable function $\psi\left(u^{1}, \ldots, u^{n}\right)$ in $\mathbf{R}_{1}^{n+1}$ and $\theta$ the hyperbolic angle between $v$ and $e_{n+1}$. If $\sinh \theta$ is bounded on $\mathbf{R}^{n}$, then $\Gamma_{\psi}$ must be a space-like hyperplane.

The following result is equivalent to Corollary 3.3.

Corollary 3.4 [15, Corollary 9.5] If an entire space-like graph $\Gamma_{\psi}$ of a $C^{2}$ differentiable function $\psi\left(u^{1}, \ldots, u^{n}\right)$ in $\mathbf{R}_{1}^{n+1}$ has constant mean curvature and there exists some constant $C(<1)$ such that $|\nabla \psi| \leq C$ on $\mathbf{R}^{n}$, then it must be a space-like hyperplane.

Indeed, if $|\nabla \psi| \leq C$ holds on $\mathbf{R}^{n}$, then we obtain

$$
\frac{|\nabla \psi|}{\sqrt{1-|\nabla \psi|^{2}}} \leq \frac{C}{\sqrt{1-C^{2}}}
$$


on $\mathbf{R}^{n}$, that is, $\sinh \theta$ is bounded because the function $t / \sqrt{1-t^{2}}$ is monotone increasing on $0<t<1$. By the same argument, the converse also holds, that is, if $\sinh \theta$ is bounded on $\mathbf{R}^{n}$, then there exists some constant $C(<1)$ such that $|\nabla \psi| \leq C$ on $\mathbf{R}^{n}$.

As described in [15, Section 9.3], Corollary 3.4 is a case of nonparametric form of the following value-distribution-theoretical property of the Gauss map.

Fact 3.5 ([16,17], [18] for a first weaker version) Let $f: M^{n} \rightarrow \mathbf{R}_{1}^{n+1}$ be a complete space-like constant mean curvature immersion. If the image of the time-like unit normal vector field (i.e., Gauss map) v( $\left.M^{n}\right)$ is contained in a geodesic ball in the hyperbolic $n$-space $\mathbf{H}^{n}(-1)$, then $f\left(M^{n}\right)$ is a space-like hyperplane in $\mathbf{R}_{1}^{n+1}$.

In fact, every entire space-like constant mean curvature graph in $\mathbf{R}_{1}^{n+1}$ is complete (see [8], note that the converse statement is also true, that is, every complete space-like hypersurface in $\mathbf{R}_{1}^{n+1}$ is an entire graph ([19])) and the image $v\left(\mathbf{R}^{n}\right)$ is contained in a geodesic ball in $\mathbf{H}^{n}(-1)$ if and only if $\sqrt{1-|\nabla \psi|^{2}}$ is bounded away from zero on $\mathbf{R}^{n}$. However the proof of Fact 3.5 is not as simple as our argument in this paper because it is obtained via an application of the Omori-Yau maximum principle [20-22]. See the book [15] for details.

For a space-like graph in $\mathbf{R}_{1}^{n+1}, \sinh \theta$ can be replaced with $1 / \sqrt{1-|\nabla \psi|^{2}}$ in (13) and (14). In fact,

$$
\sinh \theta=\frac{|\nabla \psi|}{\sqrt{1-|\nabla \psi|^{2}}}<\frac{1}{\sqrt{1-|\nabla \psi|^{2}}}
$$

holds. Thus we have the following result.

Corollary 3.6 If an entire space-like graph $\Gamma_{\psi}$ of a $C^{2}$-differentiable function $\psi\left(u^{1}, \ldots, u^{n}\right)$ in $\mathbf{R}_{1}^{n+1}$ has constant mean curvature and there exist constants $M>0$ and $\varepsilon>0$ such that

$$
\frac{1}{\sqrt{1-|\nabla \psi|^{2}}} \leq M\left(\left(u^{1}\right)^{2}+\cdots+\left(u^{n}\right)^{2}\right)^{(1 / 2)-\varepsilon}
$$

on $\mathbf{R}^{n}$, then it must be a space-like hyperplane.

From Corollary 3.6, we can show the following proposition which was given by Dong.

Proposition 3.7 ([23]) Let $\Gamma_{\psi}$ be an entire space-like constant mean curvature graph of a $C^{2}$-differentiable function $\psi\left(u^{1}, \ldots, u^{n}\right)$ in $\mathbf{R}_{1}^{n+1}$. If the function $\psi$ satisfies

$$
\frac{1}{\sqrt{1-|\nabla \psi|^{2}}}=o(r) \text { as } r \rightarrow+\infty,
$$

where $r=\sqrt{\left(u^{1}\right)^{2}+\cdots+\left(u^{n}\right)^{2}}$, then $\Gamma_{\psi}$ must be a space-like hyperplane.

Remark 3.8 In [23], Dong showed a generalization of this result for an entire spacelike graph of parallel mean curvature in the pseudo-Euclidean $(m+n)$-space $\mathbf{R}_{n}^{m+n}$ of index $n$. 


\subsection{Time-like case}

Then $|\nabla \psi|>1$ holds. By Theorem 2.1, we obtain the following Heinz-type estimate for the mean curvature of time-like graphs in $\mathbf{R}_{1}^{n+1}$.

Corollary 3.9 Let $\Gamma_{\psi}$ be a time-like graph of a $C^{2}$-differentiable function $\psi\left(u^{1}, \ldots, u^{n}\right)$ on $B^{n}(R)$ in $\mathbf{R}_{1}^{n+1}$. Suppose that there exist constants $M>0$ and $k \in \mathbf{R}$ such that

$$
\frac{|\nabla \psi|}{\sqrt{|\nabla \psi|^{2}-1}} \leq M\left(\left(u^{1}\right)^{2}+\cdots+\left(u^{n}\right)^{2}\right)^{k}
$$

on $B^{n}(R)$. If the mean curvature $H$ of $\Gamma_{\psi}$ satisfies the inequality

$$
|H| \geq \alpha>0
$$

where $\alpha$ is constant, then the following inequality holds:

$$
\alpha \leq M R^{2 k-1}
$$

Moreover, we obtain the following result from Corollary 2.4.

Corollary 3.10 If an entire time-like graph $\Gamma_{\psi}$ of a $C^{2}$-differentiable function $\psi\left(u^{1}, \ldots, u^{n}\right)$ in $\mathbf{R}_{1}^{n+1}$ has constant mean curvature $H$ and there exist constants $M>0$ and $\varepsilon>0$ such that

$$
\frac{|\nabla \psi|}{\sqrt{|\nabla \psi|^{2}-1}} \leq M\left(\left(u^{1}\right)^{2}+\cdots+\left(u^{n}\right)^{2}\right)^{(1 / 2)-\varepsilon}
$$

on $\mathbf{R}^{n}$, then it must be minimal (i.e. $H \equiv 0$ ).

We note that there exist many entire time-like minimal graphs in $\mathbf{R}_{1}^{n+1}$. In fact, for a $C^{2}$-differentiable function $h(t)$ of one variable which satisfies $h^{\prime}(t)>0$ for all $t \in \mathbf{R}$, $\psi\left(u^{1}, \cdots, u^{n}\right):=u^{n}+h\left(u^{1}\right)$ gives an entire time-like minimal graph $\Gamma_{\psi}$ in $\mathbf{R}_{1}^{n+1}$.

We do not know whether Corollary 3.10 is optimal or not. However, for example, from the case where $\varepsilon=1 / 2$ and $n=2$ in Corollary 3.10, we provide the following result.

Corollary 3.11 Let $\Gamma_{\psi}$ be an entire time-like constant mean curvature graph of a $C^{2}$ differentiable function $\psi\left(u^{1}, u^{2}\right)$ in $\mathbf{R}_{1}^{3}$. If $|\nabla \psi| / \sqrt{|\nabla \psi|^{2}-1}$ is bounded on $\mathbf{R}^{2}$, then $\Gamma_{\psi}$ is a translation surface, that is, $\psi\left(u^{1}, u^{2}\right)=h\left(u^{1}\right)+k\left(u^{2}\right)$, where $h\left(u^{1}\right)$ is a $C^{2}$-differentiable function of $u^{1}$ and $k\left(u^{2}\right)$ is a $C^{2}$-differentiable function of $u^{2}$.

The latter half of the statement of Corollary 3.11 follows from the following fact.

Fact 3.12 ([24,25]) Every entire time-like minimal graph in $\mathbf{R}_{1}^{3}$ is a translation surface. 


\subsection{Closing remark}

This paper is focused on the study of entire space-like graphs and time-like graphs in $\mathbf{R}_{1}^{n+1}$. Here we explain the recent development of the study of entire constant mean curvature graphs in $\mathbf{R}_{1}^{n+1}$. The following definitions are given in [26]. Let $\psi: \Omega \rightarrow \mathbf{R}$ be a $C^{2}$-differentiable function defined on a domain $\Omega$ in $\mathbf{R}^{n}$. A point where $|\nabla \psi|<1$ (resp. $|\nabla \psi|>1,|\nabla \psi|=1$ ) is called a space-like (resp. time-like, light-like) point of $\Gamma_{\psi}$. If $\Omega$ contains only space-like (resp. time-like) points, then $\Gamma_{\psi}$ is space-like (resp. time-like). We remark that the first fundamental form $g$ of the graph $\Gamma_{\psi}$ in $\mathbf{R}_{1}^{n+1}$ is degenerate at each light-like point. If $\Omega$ contains both space-like points and time-like points, then $\Gamma_{\psi}$ is called of mixed type. Akamine, the first author, Umehara and Yamada proved the following fact.

Fact 3.13 [26, Corollary C] An entire real analytic constant mean curvature graph in $\mathbf{R}_{1}^{n+1}$ which has no time-like points and does have a light-like point must be a light-like hyperplane.

See [26] (also [27]) for the precise definition of constant mean curvature graph which does not depend on its causal type. By Fact 3.13, we know that there exists no entire real analytic constant mean curvature graph in $\mathbf{R}_{1}^{n+1}$ which has no timelike points and does have both space-like points and light-like points although there exist many nontrivial examples of entire space-like constant mean curvature graphs. Moreover, the first and third authors, Kokubu, Umehara and Yamada [28, Remark 2.2] showed that there does not exist any real analytic nonzero constant mean curvature graph of mixed type in $\mathbf{R}_{1}^{n+1}$.

Remark 3.14 Several entire zero mean curvature graphs of mixed type in $\mathbf{R}_{1}^{3}$ were found in recent years. A systematic construction of this class is given in [29]. See [30] for details about mixed type surfaces in Lorentzian 3-manifolds.

Open Access This article is licensed under a Creative Commons Attribution 4.0 International License, which permits use, sharing, adaptation, distribution and reproduction in any medium or format, as long as you give appropriate credit to the original author(s) and the source, provide a link to the Creative Commons licence, and indicate if changes were made. The images or other third party material in this article are included in the article's Creative Commons licence, unless indicated otherwise in a credit line to the material. If material is not included in the article's Creative Commons licence and your intended use is not permitted by statutory regulation or exceeds the permitted use, you will need to obtain permission directly from the copyright holder. To view a copy of this licence, visit http://creativecommons.org/licenses/by/4.0/.

\section{References}

1. Bernstein, S.: Sur un théorème de géométrie et ses applications aux équations aux dérivées partielles du type elliptique, Comm. de la Soc. Math. de Kharkov (2éme sér.) 15, 38-45 (1915-1917)

2. Nitsche, J.C.C.: Elementary proof of Bernstein's theorem on minimal surfaces. Ann. Math. 66, 543-544 (1957)

3. Heinz, E.: Über Flächen mit eineindeutiger Projektion auf eine Ebene, deren Krümmungen durch Ungleichungen eingeschränkt sind. Math. Ann. 129, 451-454 (1955) 
4. Kenmotsu, K.: Surfaces with Constant Mean Curvature, Translated from the 2000 Japanese Original by Katsuhiro Moriya and Revised by the Author, Translations of Mathematical Monographs, vol. 221. American Mathematical Society, Providence (2003)

5. Chern, S.-S.: On the curvatures of a piece of hypersurface in euclidean space. Abh. Math. Sem. Univ. Hamburg. 29, 77-91 (1965)

6. Flanders, H.: Remark on mean curvature. J. London Math. Soc. 41, 364-366 (1966)

7. Calabi, E.: Examples of Bernstein problem for some nonlinear equations. Proc Symp. Pure Appl. Math. 15, 223-230 (1968)

8. Cheng, S.-Y., Yau, S.-T.: Maximal spacelike hypersurfaces in the Lorentz-Minkowski spaces. Ann. Math. 104, 407-419 (1976)

9. Akamine, S., Umehara, M., Yamada, K.: Improvement of the Bernstein-type theorem for space-like zero mean curvature graphs in Lorentz-Minkowski space using fluid mechanical duality. Proc. Am. Math. Soc. Ser. B 7, 17-27 (2020)

10. Bers, L.: Mathematical Aspects of Subsonic and Transonic Gas Dynamics. Wiley, New York (1958)

11. Treibergs, A.: Entire spacelike hypersurfaces of constant mean curvature in Minkowski space. Invent. Math. 66, 39-56 (1982)

12. Salavessa, I.M.C.: Spacelike graphs with parallel mean curvature. Bull. Belg. Math. Soc. Simon Stevin 15, 65-76 (2008)

13. O’Neill, B.: Semi-Riemannian Geometry with Applications to Relativity. Academic Press, London (1983)

14. López, R.: The Dirichlet problem of the constant mean curvature equation in Lorentz-Minkowski space and in Euclidean space, Mathematics (2019), 1211

15. Alías, L.J., Mastrolia, J.P., Rigoli, M.: Maximum Principles and Geometric Applications. Springer Monographs in Mathematics. Springer, Berlin (2016)

16. Aiyama, R.: On the Gauss map of complete space-like hypersurfaces of constant mean curvature in Minkowski space. Tsukuba J. Math. 16, 353-361 (1992)

17. Xin, Y.L.: On the Gauss image of a spacelike hypersurface with constant mean curvature in Minkowski space. Comment. Math. Helv. 66, 590-598 (1991)

18. Palmer, B.: The Gauss map of a spacelike constant mean curvature hypersurface of Minkowski space. Comment. Math. Helv. 65, 52-57 (1990)

19. Alías, L.J., Romero, A., Sánchez, M.: Uniqueness of complete spacelike hypersurfaces of constant mean curvature in generalized Robertson-Walker spacetimes. Gen. Relativ. Gravit. 27, 71-84 (1995)

20. Chen, Q., Xin, X.L.: A generalized maximum principle and its applications in geometry. Am. J. Math. 114, 355-366 (1992)

21. Omori, H.: Isometric immersions of Riemannian manifolds. J. Math. Soc. Jpn 95, $205-214$ (1967)

22. Yau, S.-T.: Harmonic functions on complete Riemannian manifolds. Commun. Pure Appl. Math. 28, 201-228 (1975)

23. Dong, Y.: Bernstein theorems for space-like graphs with parallel mean curvature and controlled growth. J. Geom. Phys. 58, 324-333 (2008)

24. Magid, M.A.: The Bernstein problem for timelike surfaces. Yokohama Math. J. 37, 125-137 (1990)

25. Milnor, T.K.: A conformal analog of Bernstein's theorem for timelike surfaces in Minkowski 3-space, in: The Legacy of Sonya Kovalevskaya. In: Contemp. Math. 64, 123-130 (1987)

26. Akamine, S., Honda, A., Umehara, M., Yamada, K.: Bernstein-type theorem for zero mean curvature hypersurfaces without time-like points in Lorentz-Minkowski space, to appear in Bull. Braz. Math. Soc., New Series, https://doi.org/10.1007/s00574-020-00196-8

27. Umehara, M., Yamada, K.: Hypersurfaces with light-like points in a Lorentzian manifold. J. Geom. Anal. 29, 3405-3437 (2019)

28. Honda, A., Koiso, M., Kokubu, M., Umehara, M., Yamada, K.: Mixed type surfaces with bounded mean curvature in 3-dimensional space-times. Differ. Geom. Appl. 52, 64-77 (2017)

29. Fujimori, S., Kawakami, Y., Kokubu, M., Rossman, W., Umehara, M., Yamada, K.: Entire zero-mean curvature graphs of mixed type in Lorentz-Minkowski 3-space. Q. J. Math. 67, 801-837 (2016)

30. Honda, A., Saji, K., Teramoto, K.: Mixed type surfaces with bounded Gaussian curvature in threedimensional Lorentzian manifolds. Adv. Math. 365, 107036 (2020)

Publisher's Note Springer Nature remains neutral with regard to jurisdictional claims in published maps and institutional affiliations. 